\title{
Influence of SNF1 complex on growth, glucose metabolism and mitochondrial respiration of Saccharomyces cerevisiae
}

Cecilia Martinez-Ortiz ${ }^{1}$, Andres Carrillo-Garmendia ${ }^{1}$, Blanca Flor Correa-Romero ${ }^{1}$, Melina Canizal-García ${ }^{1}$, Juan Carlos González-Hernández², Carlos RegaladoGonzalez $^{3}$, Ivanna Karina Olivares-Marin ${ }^{3}$ and Luis Alberto Madrigal-Perez ${ }^{1 *}$

${ }^{1}$ Laboratorio de Biotecnología Microbiana, Instituto Tecnológico Superior de Ciudad Hidalgo, Av. Ing. Carlos Rojas Gutiérrez \#2120, Ciudad Hidalgo, Michoacán, 61100, México.

${ }^{2}$ Laboratorio de Bioquímica del Instituto Tecnológico de Morelia, Av. Tecnológico de Morelia, Morelia, Michoacán, 58030, México.

${ }^{3}$ Universidad Autónoma de Querétaro, Cerro de las Campanas, Santiago de Querétaro, Qro, 76010, México.

* Address for correspondence:

Phone: +52 (786) 154-9000

Fax: +52 (786) 154-9000

E-mail: lmadrigal@itsch.edu.mx

\begin{abstract}
The switch of mitochondrial respiration to fermentation as the main pathway to produce ATP through the increase of glycolytic flux is known as the Crabtree effect. The elucidation of the molecular mechanism of the Crabtree effect may have important applications in ethanol production and lay the groundwork for the Warburg effect, which is essential in the molecular etiology of cancer. A key piece in this mechanism could be Snf1p, which is a protein that participates in the nutritional response that includes glucose metabolism. Thus, this work aimed to recognize the role of the SNF1 complex on the glycolytic flux and mitochondrial respiration, to gain insights about its relationship with the Crabtree effect. Herein, we found that in Saccharomyces cerevisiae cells grown at $1 \%$ glucose, mutation of SNF1 gene decreased glycolytic flux, increased $\mathrm{NAD}(\mathrm{P}) \mathrm{H}$, enhanced $H X K 2$ gene transcription, and decreased mitochondrial respiration. Meanwhile, the same mutation increased the mitochondrial respiration of cells grown at $10 \%$ glucose. Moreover, SNF4 gene deletion increased respiration and growth at $1 \%$ of glucose. In the case of the GAL83 gene, we did not detect any change in mitochondrial-respiration or growth. Altogether, these findings indicate that $S N F 1$ is vital to switch from mitochondrial respiration to fermentation.
\end{abstract}

Keywords: Crabtree effect; glycolytic flux; mitochondrial respiration; Saccharomyces cerevisiae; Snf1p; fermentation. 


\section{Introduction}

One of the most relevant metabolic capacities of Saccharomyces cerevisiae is the ability to grow under aerobic and anoxic conditions; this yeast has evolved to use complex mechanisms to obtain energy in both circumstances switching the metabolism from respiration to mainly fermentation. The amount of fermentable sugars in the medium also promotes the preference in energy production of $S$. cerevisiae. Fermentable sugars concentration $>0.8 \mathrm{mM}$ favors energy generation through fermentation, while lower levels stimulate mitochondrial respiration (De Deken, 1966, Hagman, et al., 2014). Interestingly, when glucose availability is enough, the yeast prefers the fermentative metabolism even in the presence of oxygen, and represses mitochondrial respiration, this phenotype is known as the Crabtree effect (Moriya \& Johnston, 2004). Nonetheless, the metabolic regulation that governs these changes is not entirely understood. The increase of fermentable sugars availability inactivates the sucrose non-fermenting protein-1 (Snflp) suggesting that this protein plays a vital role in establishing fermentation as the primary source of ATP production (Kayikci \& Nielsen, 2015) (Celenza \& Carlson, 1984).

The Snflp protein is orthologous to the mammalian AMP-kinase (AMPK) (Hedbacker \& Carlson, 2008). Snf1p is a catabolic regulator that could be activated by increasing the ADP/ATP ratio (Mayer, et al., 2011), or through phosphorylation by the kinases Sak1p, Elm3p, and Tos3p (Hong, et al., 2003, Kim, et al., 2005, Liu, et al., 2011). Snflp activation has been linked to several catabolic pathways regulation such as mitochondrial respiration (Wright \& Poyton, 1990), fatty acid metabolism (Zhang, et al., 2013), and glycolysis (Nicastro, et al., 2015). However, modulation of Snflp is intimately related to the glycolysis function. In this regard, the mutation of SNF1 gene has profound effects on glucose metabolism. For example, the snfl $\triangle$ mutant strain is unable of growing in media with low levels of glucose, but at high levels, the growth is similar to the wild type strains (Carlson, et al., 1981). Additionally, the glycolytic flux is also impaired by deletion of the SNF1 gene (Nicastro, et al., 2015). A key player in the phenotype showed by the $s n f l \Delta$ mutant is Mig1p, which is phosphorylated and inhibited by Snf1p and is responsible for regulating several proteins including the hexose transporters HXT1 and HXT3. Accordingly, the MIGl mutation partially relieves the glucose repression (Treitel, et al., 1998). Interestingly, the snfl $\Delta$ mutant strain is more susceptible to the toxicity induced by deoxyglucose (glucose analog that inhibits glycolysis), and the overexpression of the Hxt1p and Hxt3p release the mutant strain from this toxicity (O'Donnell, et al., 2015). One of the most accepted theories about the Crabtree effect is that it originates by the hexose transporters modulated by the glycolytic flux (Huberts, et al., 2012).

Thereby the glucose repression might be originated by the down-regulation of specific hexose transporters through the Snf1p/Mig1p pathway. Hence, our work aimed to determine whether deletion of the $S N F 1$ gene in $S$. cerevisiae impairs the glycolytic flow and how it affects the switch of mitochondrial respiration to fermentation due to glucose repression.

\section{Material and methods}

Strains

The experiments were carried out in the genetic background of S. cerevisiae BY4742 
(MaTa, his3 $\triangle 1$, leu2 $\triangle 0$, lys2 $\triangle 0$, ura3 $\triangle 0$ ) and its mutant in the genes SNF1 (snfl ,

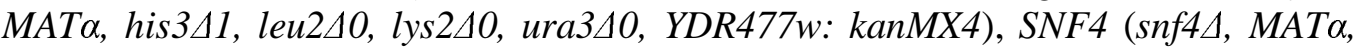
his3A1, leu2A0, lys2A0, ura3A0, YGL115w: kanMX4), GAL83 (gal83A, MATa, his3A1, leu2A0, lys2 $\triangle 0$, ura3 $\triangle 0$, YER027c: kanMX4) acquired from the EUROSCARF program (University of Frankfurt, Germany). Strains were maintained in YPD medium (1\% yeast extract, $2 \%$ casein peptone and $2 \%$ glucose). The mutant strains were supplemented with the antibiotic geneticin $(200 \mathrm{mg} / \mathrm{mL})(\mathrm{G}-418$, SigmaAldrich, St. Louis, MO, USA).

\section{Experimental design}

To verify that Snflp is mainly regulated by the concentration of carbon sources and not by nitrogen sources, we used a $3^{4}$ full factorial randomized design. The following factors were evaluated: type of carbon source (levels: glucose, sucrose and galactose), type of nitrogen source (levels: proline, glutamate, and ammonium sulfate), carbon concentration (levels (w/v): $0.01 \%, 2 \%$, and $10 \%$ ), and nitrogen concentration (levels (w/v): $0.01 \%, 0.5 \%$ and $5 \%$ ). A total of 81 nutritional conditions were assayed three to five times with two technical replicates. The same experimental design was performed using the BY4742 and snfl $\Delta$ strains separately. The response variable was the percentages of growth (specific growth rate) of the mutant snflA relative to the growth of WT multiplied by 100. Cultures were grown on honeycomb plates (Growth Curves, Piscataway, NJ, USA) with $145 \mu \mathrm{L}$ of medium per well. Each well was inoculated with $5 \mu \mathrm{L}$ of an overnight $S$. cerevisiae BY4742 or snfl $\Delta$ culture, grown in YPD medium at $30{ }^{\circ} \mathrm{C}$ in an orbital shaker (MaxQ 6,000, Thermo Scientific, Waltham, MA, USA) at $250 \mathrm{rpm}$. Samples were incubated in $\mathrm{SC}$ medium at $30{ }^{\circ} \mathrm{C}$ for $48 \mathrm{~h}$ using a Bioscreen (model C MBR, Growth Curves) programmed with continuous shaking at medium speed and readings at $600 \mathrm{~nm}$, every $30 \mathrm{~min}$. A dendogram was constructed using the average linkage clustering method and the Euclidean distance measurement method using the program heatmapper (Babicki, et al., 2016).

\section{Plate serial dilutions}

Each strain was grown in $3 \mathrm{~mL}$ of YPD medium supplemented with $2 \%$ glucose in 10 $\mathrm{mL}$ test tubes inoculated with three single colonies from YPD agar plates. The cells were kept in the incubator with constant shaking $(200 \mathrm{rpm})$ for 18 hours at $30{ }^{\circ} \mathrm{C}$. The inoculum optical density at $600 \mathrm{~nm}\left(\mathrm{OD}_{600}\right)$ was adjusted to a final value of $\sim 0.3$ for serial dilutions. Then, $3 \mu \mathrm{L}$ of 10 -fold serial dilutions were plated on YPD medium supplemented with two concentrations of glucose ( 1 and $0.1 \%)$. Plates were incubated at $30{ }^{\circ} \mathrm{C}$ for $48 \mathrm{~h}$.

\section{Extracellular acidification rate}

A $3 \mathrm{~mL}$ YPD medium was inoculated with a fresh colony grown on the YPD agar plates and cultured at $30{ }^{\circ} \mathrm{C}$ and $200 \mathrm{rpm}$ overnight. The pre-culture was used to inoculate $250 \mathrm{~mL}$ flasks with $50 \mathrm{~mL}$ of YPD medium supplemented with 1 or $10 \%$ glucose. The cells were harvested at mid-log phase $\left(\mathrm{OD}_{600} \sim 0.5\right)$ at $9,000 \mathrm{~g}$ for 5 minutes, and three washes were performed with sterile water, and the cell pellet was resuspended in $2 \mathrm{~mL}$ of sterile water. Then, cells were added to $25 \mathrm{~mL}$ of distilled water to obtain a final concentration of about $120 \mathrm{mg}$ cells $/ \mathrm{mL}$. The extracellular acidification rate experiment was initiated by adding $1 \mathrm{~mL}$ of $1 \mathrm{M}$ glucose, and recording the $\mathrm{pH}$ in the Metrohm 902 Titrando equipment (Metrohm, FL, USA), fitted with the Tiamo software version 2.3 for 5 minutes. Data were analyzed with the GraphPad Prism 6.00 for Macintosh (GraphPad Software, La Jolla California, USA). 
Quantification of NADH/NAD ${ }^{+}$

Cells were cultured in $10 \mathrm{~mL}$ of YPD medium supplemented with $1 \%$ or $10 \%(\mathrm{w} / \mathrm{v})$ glucose at $30{ }^{\circ} \mathrm{C}$ with constant agitation $(200 \mathrm{rpm})$, when cells reached the mid-log phase $\left(\mathrm{OD}_{600} \sim 0.5\right) 35 \mathrm{~mL}$ of methanol was added. Then, the solution was centrifuged at $4,000 \mathrm{~g}$ for 1 minute at $-20{ }^{\circ} \mathrm{C}$, immediately resuspended in $0.25 \mathrm{~mL}$ of $0.1 \mathrm{~N}$ $\mathrm{NaOH}(\mathrm{NADH})$ or $0.1 \mathrm{~N} \mathrm{HCl}\left(\mathrm{NAD}^{+}\right)$, boiled for 1 minute and centrifuged at 5,000 $g$ for 5 minutes to separate and discard the cellular debris. Concentrations of NADH and $\mathrm{NAD}^{+}$were determined according to Bernofsky and Swan (1973).

\section{$R T-q P C R$}

The strains were grown in $3 \mathrm{~mL}$ of YPD medium supplemented with 1 or $10 \%$ glucose until the mid-log phase $\left(\mathrm{OD}_{600} \sim .5\right)$ was reached. Subsequently, the cells were harvested to remove the culture medium, centrifuging at $13,000 \mathrm{~g}$ for 3 minutes. Then, $0.75 \mathrm{~mL}$ of TRIzol (Ambion, Life Technologies, Foster City, CA, USA) was added (the cell pellet was not washed with TRIzol to avoid lysis and release of nucleases), homogenized at 3,000 rpm for 15 seconds, and immediately placed in ice. The suspension was centrifuged at $12,000 \mathrm{~g}$ for 10 minutes at $4{ }^{\circ} \mathrm{C}$, and the supernatant was transferred to a new microtube, followed by incubation for 5 minutes at room temperature, to allow complete dissociation of the nucleoprotein complexes. Later, $0.2 \mathrm{~mL}$ of cold chloroform was added per $\mathrm{mL}$ of TRIzol, hand shaken vigorously for 15 seconds and incubated for 2-3 minutes at room temperature, followed by centrifugation at $12,000 \mathrm{~g}$ for 15 minutes at $4{ }^{\circ} \mathrm{C}$. The aqueous phase was immediately transferred to a sterile microtube where $0.5 \mathrm{~mL}$ of $100 \%$ isopropanol was added and incubated for 10 minutes, before centrifuging at 12,000 $\mathrm{g}$ for 10 minutes at $4{ }^{\circ} \mathrm{C}$, and the supernatant was removed. The pellet was washed with $1 \mathrm{~mL}$ of $75 \%$ ethanol, homogenized and then centrifuged at 7,500 $\mathrm{g}$ for 5 minutes at $4{ }^{\circ} \mathrm{C}$. The pellet was allowed to dry, and then $50 \mu \mathrm{L}$ diethylpyrocarbonate water (DEPC), and recombinant DNAase I, RNAse free (Roche Diagnostics GmbH, Manheim, Germany) were added. The integrity of the RNA was verified with an electrophoretic analysis, as well as the concentration and quality of the RNA using a Nanodrop 2000 (ThermoScientific, Whaltham, MA, USA).

To carry out the qPCR, the complementary DNA synthesis (cDNA) was performed. We used $2.5 \mu \mathrm{g}$ of total RNA and the RevertAid H Minus first chain cDNA synthesis kit (Thermo-Scientific). First, $1 \mu \mathrm{L}$ of random hexamer primer $(200 \mathrm{ng}), 12 \mu \mathrm{L}$ of DEPC water and $1 \mu \mathrm{L}$ of dNTP $(10 \mathrm{mM})$ were added. It was mixed and heated at 65 ${ }^{\circ} \mathrm{C}$ for 5 minutes, allowed to cool on ice, added with $4 \mu \mathrm{L}$ of $5 \mathrm{x}$ buffer, and $2 \mu \mathrm{L}$ of DTT $(0.1 \mathrm{M})$, mixed and heated at $37{ }^{\circ} \mathrm{C}$ for 2 minutes. Then $1 \mu \mathrm{L}$ of RevertAid $\mathrm{H}$ Minus M-MuLV RT was added, allowed to incubate at room temperature for 10 minutes. Then, it was heated at $37{ }^{\circ} \mathrm{C}$ for 50 minutes and finally heated at $70{ }^{\circ} \mathrm{C}$ for 15 minutes. Once the cDNA was synthesized, the RT-qPCR was continued. Each reaction had a final volume of $20 \mu \mathrm{L}, 2 \mu \mathrm{L}$ of cDNA, $10 \mu \mathrm{L}$ of SYBR Select Master Mix (AppliedBiosystems), $0.8 \mu \mathrm{L}$ of each primer and $6.4 \mu \mathrm{L}$ of DEPC water were added. Subsequently, they were placed in the Rotor-Gene Q (Qiagen, Venlo, The Netherlands).

The genes that were evaluated at the transcriptional level were $H X K 2$ and $P F K 1$, using as a reference gene UBC6 (ubiquitin-conjugation-6). Conditions used to carry out the qPCR were those depicted by the protocol of Madrigal-Perez, et al. (2015) 
which were: beginning with the activation of UDG at $50{ }^{\circ} \mathrm{C}$ for 2 minutes, followed by the activation of AmpliTaq-DNA at $95{ }^{\circ} \mathrm{C}$ for 2 minutes with 35 cycles of denaturation at $94{ }^{\circ} \mathrm{C}$ for 15 seconds. The alignment temperature of oligos $H X K 2$ and UBC6 was $51{ }^{\circ} \mathrm{C}$, oligo PFK1 was $54{ }^{\circ} \mathrm{C}$ for 30 seconds, and the extension temperature of $72{ }^{\circ} \mathrm{C}$ for 1 minute, followed by 1 cycle at $72{ }^{\circ} \mathrm{C}$ for 7 minutes.

\section{Determination of in situ mitochondrial respiration}

The mitochondrial respiration was performed according to Tello-Padilla, et al. (2017). Briefly, the strains were cultured in $50 \mathrm{~mL}$ of YPD medium supplemented with (w/v) $1 \%$ or $10 \%$ glucose at $30{ }^{\circ} \mathrm{C}$ and $200 \mathrm{rpm}$ until the mid-log phase $\left(\mathrm{OD}_{600} \sim 0.5\right)$ was reached. Cells were harvested at $5,000 \mathrm{~g}$ for 5 minutes, and three washes were performed with deionized water and resuspended in a 1:1 ratio $(\mathrm{w} / \mathrm{v})$. Oxygen consumption was analyzed polarographically with a Clark type electrode connected to a YSI5300A monitor (Yellow Springs, OH, USA) and a computer for data acquisition. In the polarograph chamber $125 \mathrm{mg}$ of cells (wet weight), $5 \mathrm{~mL}$ of MESTEA buffer (10 mM morphoethanolsulfonic acid, $\mathrm{pH} 6.0$ with triethanolamine) and glucose $(10 \mathrm{mM})$ were added; the cells were maintained with constant agitation (basal respiration). Subsequently, the uncoupled state (maximum respiration) was stimulated with the addition of $0.015 \mathrm{mM}$ carbonylcyanide- $p$-trichlorophenylhydrazone (CCCP) for 3 minutes. Then, electron transport chain (ETC) inhibitors were added: thenoyltrifluoroacetone (TTFA, $1 \mathrm{mM}$ ), antimycin A (AA) and $0.75 \mathrm{mM} \mathrm{KCN}$ (nonmitochondrial respiration), each inhibitor was left for 3 minutes. The results were analyzed using the statistical package GraphPad Prism 6.00 for Macintosh (GraphPad Software).

\section{Results}

Effect of the chemical nature and concentration of carbon and nitrogen in growth of the mutant $\operatorname{snf} 1 \Delta$

To verify that Snflp is mainly regulated by the concentration of carbon sources and not by nitrogen sources, we made an experimental design with three concentrations of nitrogen and carbon sources using three different carbon sources (galactose, glucose, and sucrose) and three nitrogen sources (proline, ammonium, and glutamate). We found that the main factor affecting the growth of the mutant snfl $\Delta$ was the carbon source concentration (Fig. 1). However, the nitrogen source and its concentration do not show any effect upon the growth of the mutant snflA (Fig. 1). Growth decrease is evident at $\geq 0.5 \%$ glucose (Fig. 1). These data corroborate the critical role of SNF1 gene in the growth of $S$. cerevisiae under low concentration of carbon sources.

Impact of the SNF1 mutation in the glycolytic flux

To obtain the first insights about how the SNF1 gene affects glucose metabolism, we decided to measure the extracellular acidification rate as an indicator of the glycolytic flux. Two glucose concentrations were used (1\% and 10\%) since concentrations below $0.5 \%$ glucose the mutant $s n f 1 \Delta$ showed a negligible growth. Interestingly, at $1 \%$ glucose the mutant snflA showed a lower acidification rate than the WT strain (Fig. 2), while at $10 \%$ glucose no differences were revealed (Fig. 2). These data proved that the SNF1 gene is vital to sustain the glycolytic flux in a low glucose concentration.

\section{Influence of $\mathrm{SNF} 1$ mutation on $N A D H / N A D^{+}$and $N A D(P) H$ ratios}

A useful indicator of how glycolysis is operating $\mathrm{NADH} / \mathrm{NAD}^{+}$ratio since it is a key 
regulator of this pathway. This parameter was measured, to discard whether the decrease of the glycolytic flux is due to a down-regulation of the glycolysis pathway. No differences were found either at $1 \%$ or $10 \%$ of glucose in the NADH/NAD ${ }^{+}$ratio (Fig. 3). Another possibility is that glucose-6-phosphate has been metabolized by the pentose phosphate pathway, to prove this idea the $\mathrm{NAD}(\mathrm{P}) \mathrm{H}$ was measured, since we did not find any difference in the $\mathrm{NADH} / \mathrm{NAD}^{+}$ratio. Therefore glycolysis changes could be attributed to NADPH variations. At $1 \%$ glucose, we found higher $\mathrm{NAD}(\mathrm{P}) \mathrm{H}$ concentration in the mutant snfld than in the WT strain (Fig. 3). However, no differences were observed at $10 \%$ glucose. These data suggest that a decrease of the glycolytic flux of the mutant snfl $1 \Delta$ could be due to a bypass of glycolysis to the pentose phosphate pathway at $1 \%$ glucose.

SNF1 deletion affects the transcription of the HXK2 and PFK1 genes

Mutation of the SNF1 gene could affect the transcription of $H X K 2$ and $P F K 1$, two critical genes of the glycolysis pathway that in turn possibly results in the glycolytic flux impairment. Regarding the $H X K 2$ gene, it was found that transcript levels in the mutant snfl $1 \Delta$ were higher than in the WT strain at $1 \%$ glucose; while no differences were found at $10 \%$ glucose (Fig. 4). PFK1 transcript levels did not change under any condition (Fig. 4). These data indicate that higher transcription levels of the HXK2 gene could be related to the decrease of glycolytic flux seen in the mutant $\operatorname{snf} 1 \Delta$ at $1 \%$ glucose.

\section{Effect of SNF1 mutation in the mitochondrial respiration}

The glycolytic flux is tightly coupled with mitochondrial respiration in $S$. cerevisiae. For example, the Crabtree effect is characterized by a decrease of the mitochondrial respiration and an increase of the glycolytic flux. To further understand the role of the gene SNF1 in this phenotype, the mitochondrial respiration was measured. Interestingly, basal respiration and the maximal respiratory capacity were reduced in the mutant snflA at $1 \%$ glucose in comparison with the WT strain (Fig. 5). Surprisingly, at $10 \%$ glucose the opposite phenotype was seen, the basal respiration and the maximal respiratory capacity were higher in the mutant snfl $\triangle$ than in the WT strain (Fig. 5). These data indicate that mitochondrial respiration is affected by $S N F 1$ mutation in a glucose-dependent manner.

\section{Influence of the SNF1 complex in the mitochondrial respiration}

Snf1p is part of the SNF1 complex, which structurally is a heterotrimer consisting of the catalytic subunit $\operatorname{Snf} 1 p(\alpha)$, the regulatory subunit $\operatorname{Snf} 4 p(\gamma)$ and three alternative subunits Gal83p, Sip1p and Sip2p ( $\beta$ ). It has been shown that Snf4p binds to the regulatory domain in the Snf1p protein preventing the autoinhibition of the kinase activity of Snf1p under low glucose concentrations (Jiang \& Carlson, 1996). The regulatory subunits $\operatorname{Snf} 4 \mathrm{p}(\gamma)$ and $\operatorname{Gal} 83 \mathrm{p}(\beta)$ have crucial participation in the activation and correct function of Snf1p. To understand the participation of the SNF1 complex in the mutants snf4A and gal83A the mitochondrial respiration was measured. Snf4p prevents the inhibition of Snf1p, and based on this, it was expected that mutant $s n f 4 \Delta$ shows a phenotype similar to that of snfl $1 \Delta$. However, at $1 \%$ glucose the mutant snf4 $\Delta$ exhibited higher mitochondrial respiration than the WT strain, and this phenotype is also seen in $10 \%$ glucose (Fig. 6). On the contrary, the GAL83 deletion has no impact on mitochondrial respiration (Fig. 6). Interestingly, deletion of SNF4 or GAL83 resulted in increased growth of $S$. cerevisiae even at low glucose concentration $(0.1 \%)$ (Fig. 7). These data suggest that Snf1p has a specific role in 
growth and mitochondrial respiration of $S$. cerevisiae, independent of the SNF1 complex.

\section{Discussion}

The molecular mechanism behind the Crabtree effect remains largely elusive. Control and regulation of this phenotype could help improve ethanol production and establish the basis for the Warburg effect, which is essential in the molecular etiology of cancer. The inhibition of Snf1p by glucose repression indicates that this protein may regulate the Crabtree effect. However, a clear relationship between the Crabtree effect and Snf1p has not been found yet. Cells glucose uptake is the main factor associated with the Crabtree effect and is regulated by hexose transporters mainly, and in turn the Snf1p/Mig1p pathway regulates some hexose transporters transcriptionally. These data imply that Snflp could regulate the glycolytic flux and the Crabtree effect, and our results support this idea. The SNF1 mutant is unable to grow in media with low concentration of carbon sources. At $1 \%$ glucose, the mutant snfl $\triangle$ showed a decrease in the glycolytic flux, increased $\mathrm{NAD}(\mathrm{P}) \mathrm{H}$ concentration, enlarged transcription of the $H X K 2$ gene and reduced mitochondrial respiration. However, the SNF1 deletion keeps the $\mathrm{NADH} / \mathrm{NAD}^{+}$ratio, and the $\mathrm{NAD}(\mathrm{P}) \mathrm{H}$ concentration constant. Also the transcription of genes $H X K 2$ and $P F K 1$ at $10 \%$ glucose did not show any change, but only the mitochondrial respiration increased. Although the SNF4 deletion maintains Snf1p inhibited, the phenotypes observed in mitochondrial respiration and growth were different from the SNF1 mutant, associated to an increase at $1 \%$ glucose. In the case of the GAL83, we did not detect any change in the mitochondrial respiration or growth. Altogether these findings indicate that SNF1 is important in the switch of mitochondrial respiration to fermentation.

The signal pathway governed by Snf1p is responsible for the reshaping of the cell metabolism to obtain energy during starvation or in a low-energy environment. In this sense, Snf1p responds primarily to carbon availability and its concentration, irrespective of other carbon or nitrogen sources in the media. It is important to note that concentration of the carbon source is the key factor in SNF1 mutant growth. This phenotype was also observed in a glucose-repressible carbon source, such as galactose whose canonical regulation pathway is modulated by the Snflp/Mig1p pathway. This particular phenotype could be explained by the fact that galactose uptake and metabolism are not exclusively regulated by the Snf1p/Mig1p pathway, but also by its proportion relative to glucose (Escalante-Chong, et al., 2015). It has been shown that the extracellular glucose modulates the glucose transporters at a transcription level (Ozcan \& Johnston, 1999, Reijenga, et al., 2001). Moreover, the signaling pathway Snf1p/Mig1p repressed high-affinity transporters at high glucose levels. On the contrary, when Snflp is activated at low glucose concentration, the HXT6 and HXT7 genes are de-repressed. Transport of the carbon source is the primary regulator of the glycolytic flux; these data indicate that reduced growth of the SNF1 mutant could be due to impairment of the glycolytic flux function. Interestingly, the glycolytic flux is only disturbed when the SNF1 mutant was grown in $1 \%$ glucose, and no changes were observed at $10 \%$ glucose. This phenotype highlights the importance of $S N F 1$ at low glucose concentrations. However, it was also reported that at $2 \%$ glucose, $S N F 1$ mutant grown in synthetic medium showed similar glucose consumption as the WT strain (BY4741), but glucose consumption increased at $5 \%$ concentration, relative to the WT strain (Nicastro, et al., 2015). Differences from previous reports could due to the different medium used. 
Nevertheless, it was observed that snflA cells produced fermentation by-products other than ethanol and acetate when grown at $2 \%$ glucose and the same amounts were produced in $10 \%$ of glucose, when compared to the WT strain (Nicastro, et al., 2015). We did not find any difference in the NADH/NAD ${ }^{+}$ratio when the WT and snflA cells were grown in $1 \%$ and $10 \%$ glucose. To discard changes in the NADPH species we measured the $\mathrm{NAD}(\mathrm{P}) \mathrm{H}$ fluorescence, detecting an increased concentration in the snfld mutant at $1 \%$ glucose, whereas no difference was detected at $10 \%$ glucose. According to these data NADPH increased in the mutant growing in $1 \%$ of glucose. To gain insights into this change the transcription of two key genes in the glycolysis pathway, $H X K 2$, and PFK1 was evaluated. An increase in $H X K 2$ transcription at $1 \%$ glucose in snfl $1 \triangle$ cells was the only change found. The increased levels of NADPH and $H X K 2$ gene transcription suggests that high quantities of glucose-6-phosphate are produced and metabolized via the pentose phosphate pathway. A report supporting this assumption measured the expression of two low-affinity glucose transporters (Hxt1p and the recombinant TMO) in a $S$. cerevisiae strain of without hexose transporters. They found increased concentration of glucose-6-phosphate, and fructose-6-phosphate and decreased levels of fructose-1,6-biphosphate, those concentrations might inhibit the activity of the Pfk1p enzyme (Bosch, et al., 2008). Remarkably, the recombinant strains that express the low-affinity glucose transporters experience a lessening in the consumption rate of glucose and fructose, and show a fully respiratory metabolism (Bosch, et al., 2008). In this study we found that mitochondrial respiration decreased in the snfl 14 mutant growing in $1 \%$ glucose, while at $10 \%$ glucose the growth was higher than that exhibited by the WT. The increased mitochondrial respiration at $10 \%$ glucose clearly showed the importance of SNF1 in the switch of mitochondrial respiration to fermentation to obtain ATP. At 10\% glucose Snf1p is inhibited by the glucose repression, so we expected that at this concentration the SNF1 deletion showed no metabolic effect according to all parameters assayed. However, the fact that SNF1 mutation increased mitochondrial respiration even at high glucose concentration suggests the important function of this gene even in high concentrations of this carbohydrate. In this regard, it has been proposed that at high glucose concentrations Snf1p could form complexes, which in turn would have activated a different set of transcriptional factors implicated in the adaptation of cells growth in fermentation conditions. This phenotype is evident in the transcriptional regulation of the $T P K 1$ gene by the Snf1p/Cat8p pathway (Galello, et al., 2017). Another example of this model is the endocytosis of the high-affinity glucose transporter Hxt6p at high glucose concentrations that requires the arrestingrelated trafficking adaptor Rod1p/Art4p, whose function is regulated by $\mathrm{Snf} 1 \mathrm{p}$ (Llopis-Torregrosa, et al., 2016). On the contrary, the decrease in mitochondrial respiration observed at $1 \%$ glucose could be related to reduction of the glycolytic flux and cellular viability in the mutant snfl $1 \triangle$. Altogether, these results indicate that SNF1 gene is important to modulate the glycolytic flux and mitochondrial respiration, which is essential at low carbon sources concentration, whereas at higher glucose concentrations SNF1 gene is critical for the establishment of fermentation.

Finally, these findings suggest that SNF1 controls the glycolytic flux and mitochondrial respiration through regulation of the hexose transporters, which in turn allow switching from mitochondrial respiration to fermentation.

\section{Funding}

This work was supported by grants from Instituto Tecnológico Superior de Ciudad 
Hidalgo (3308.100310), Tecnológico Nacional de México (165.14.2-PD and 166.14.2-PD) and PROMEP program.

\section{Acknowledgments}

The authors would like to thank Minerva Ramos-Gomez for their technical support. The authors declare no competing financial interest.

\section{References}

Babicki S, Arndt D, Marcu A, Liang Y, Grant JR, Maciejewski A \& Wishart DS (2016) Heatmapper: web-enabled heat mapping for all. Nucleic Acids Res. 8: W147153.

Bernofsky C \& Swan M (1973) An Improved Cycling Assay for Nicotinamide Adenine Dinucleotide. Analytical Biochemistry 53: 452-458.

Bosch D, Johansson M, Ferndahl C, Franzen CJ, Larsson C \& Gustafsson L (2008) Characterization of glucose transport mutants of Saccharomyces cerevisiae during a nutritional upshift reveals a correlation between metabolite levels and glycolytic flux. FEMS Yeast Res 8: 10-25.

Carlson M, Osmond BC \& Botstein D (1981) Mutants of yeast defective in sucrose utilization. Genetics 98: 25-40.

Celenza JL \& Carlson M (1984) Cloning and Genetic Mapping of SNF1, a Gene Required for Expression of Glucose-Represible Genes in Saccharomyces cerevisiae. Mol Cell Biol 4: 49-53.

De Deken RH (1966) The Crabtree effect: a regulatory system in yeast. J Gen Microbiol. 44: 149-156.

Escalante-Chong R, Savir Y, Carroll SM, Ingraham JB, Wang J, Marx CJ \& Springer M (2015) Galactose metabolic genes in yeast respond to a ratio of galactose and glucose. Proc Natl Acad Sci U S A. 112: 1636-1641.

Galello F, Pautasso C, Reca S, Canonero L, Portela P, Moreno S \& Rossi S (2017) Transcriptional regulation of the protein kinase a subunits in Saccharomyces cerevisiae during fermentative growth. Yeast 34: 495-508.

Hagman A, Sall T \& Piskur J (2014) Analysis of the yeast short-term Crabtree effect and its origin. FEBS J 281: 4805-4814.

Hedbacker K \& Carlson M (2008) SNF1/AMPK pathways in yeast. Front Biosci. 13: 2408-2420.

Hong SP, Leiper FC, Woods A, Carling D \& Carlson M (2003) Activation of yeast Snf1 and mammalian AMP-activated protein kinase by upstream kinases. Proc Natl Acad Sci U S A 100: 8839-8843.

Huberts DH, Niebel B \& Heinemann M (2012) A flux-sensing mechanism could regulate the switch between respiration and fermentation. FEMS Yeast Res 12: 118128.

Jiang R \& Carlson M (1996) Glucose regulates protein interactions within the yeast SNF1 protein kinase complex. Genes Dev. 10: 3105-3115.

Kayikci O \& Nielsen J (2015) Glucose repression in Saccharomyces cerevisiae. FEMS Yeast Res 15.

Kim MD, Hong SP \& Carlson M (2005) Role of Tos3, a Snf1 protein kinase kinase, during growth of Saccharomyces cerevisiae on nonfermentable carbon sources. Eukaryot Cell 4: 861-866.

Liu Y, Xu X \& Carlson M (2011) Interaction of SNF1 protein kinase with its activating kinase Sak1. Eukaryot Cell 10: 313-319. 
Llopis-Torregrosa V, Ferri-Blazquez A, Adam-Artigues A, Deffontaines E, van Heusden GP \& Yenush L (2016) Regulation of the Yeast Hxt6 Hexose Transporter by the Rod1 alpha-Arrestin, the Snf1 Protein Kinase, and the Bmh2 14-3-3 Protein. $J$ Biol Chem 291: 14973-14985.

Madrigal-Perez LA, Nava GM, Gonzalez-Hernandez JC \& Ramos-Gomez M (2015) Resveratrol increases glycolytic flux in Saccharomyces cerevisiae via a SNF1dependet mechanism. J Bioenerg Biomembr 47: 331-336.

Mayer FV, Heath R, Underwood E, et al. (2011) ADP regulates SNF1, the Saccharomyces cerevisiae homolog of AMP-activated protein kinase. Cell Metab 14: 707-714.

Moriya H \& Johnston M (2004) Glucose sensing and signaling in Saccharomyces cerevisiae through the Rgt2 glucose sensor and casein kinase I. Proc Natl Acad Sci U $S$ A 101: 1572-1577.

Nicastro R, Tripodi F, Guzzi C, et al. (2015) Enhanced amino acid utilization sustains growth of cells lacking Snf1/AMPK. Biochim Biophys Acta 1853: 1615-1625.

O'Donnell AF, McCartney RR, Chandrashekarappa DG, Zhang BB, Thorner J \& Schmidt MC (2015) 2-Deoxyglucose impairs Saccharomyces cerevisiae growth by stimulating Snf1-regulated and alpha-arrestin-mediated trafficking of hexose transporters 1 and 3. Mol Cell Biol 35: 939-955.

Ozcan S \& Johnston M (1999) Function and Regulation of Yeast Hexose Transporters. Microbiol Mol Biol Rev. 63: 554-569.

Reijenga KA, Snoep JL, Diderich JA, van Verseveld HW, Westerhoff HV \& Teusink B (2001) Control of glycolytic dynamics by hexose transport in Saccharomyces cerevisiae. Biophys J. 80: 626-634.

Tello-Padilla MF, Perez-Gonzalez AY, Canizal-Garcia M, Gonzalez-Hernandez JC, Cortes-Rojo C, Olivares-Marin IK \& Madrigal-Perez LA (2017) Glutathione levels influence chronological life span of Saccharomyces cerevisiae in a glucose-dependent manner. Yeast.

Treitel MA, Kuchin S \& Carlson M (1998) Snf1 protein kinase regulates phosphorylation of the Mig1 repressor in Saccharomyces cerevisiae. Mol Cell Biol. 18.

Wright RM \& Poyton RO (1990) Release of two Saccharomyces cerevisiae cytochrome genes, COX6 and CYC1, from glucose repression requires the SNF1 and SSN6 gene products. Mol Cell Biol. 10: 1297-1300.

Zhang M, Galdieri L \& Vancura A (2013) The yeast AMPK homolog SNF1 regulates acetyl coenzyme A homeostasis and histone acetylation. Mol Cell Biol 33: 4701-4717.

\section{Figure legends}

Figure 1. Influence of chemical nature and concentration of carbon and nitrogen sources on growth of the mutant snflA. Data in the heat map shows the cluster membership of the mutant snfl $1 \Delta$ growth relative to the WT strain times 100 . Glucose supplementation at $0.01 \%, 2 \%$, and $10 \%$ are represented by $\mathrm{X} 1, \mathrm{X} 2$, and $\mathrm{X} 3$. Galactose supplementation at $0.01 \%, 2 \%$, and $10 \%$ are represented by X4, X5, and X6. Sucrose supplementation at $0.01 \%, 2 \%$, and $10 \%$ are represented by X7, X8 and X9. Proline supplementation at $0.01 \%, 0.5 \%$, and $5 \%$ are represented by 1,2 , and 3 . Glutamate supplementation at $0.01 \%, 0.5 \%$, and $5 \%$ are represented by 4,5 , and 6 . Ammonium sulfate supplementation at $0.01 \%, 0.5 \%$, and $5 \%$ are represented by 7,8 , and 9. The average linkage clustering method was used, while Euclidean distance measurement method was used. The results represent mean values from 3-5 independent experiments, which includes mean values of 3 technical repetitions. 
Figure 2. Effect of the SNF1 deletion in the extracellular acidification rate. Media acidification was used as an indirect indicator of glucose consumption at $1 \%$ and $10 \%$ glucose. The results represent mean values \pm SEM from 3-4 independent experiments. Statistical analyses were performed using two-tailed paired Student $t$-test.

Figure 3. Variation of the $\mathrm{NADH} / \mathrm{NAD}^{+}$ratio and $\mathrm{NAD}(\mathrm{P}) \mathrm{H}$ in the mutant snflA. $\mathrm{NADH} / \mathrm{NAD}^{+}$ratio measurement was performed using a cycling assay. NAD $(\mathrm{P}) \mathrm{H}$ was calculated by fluorescence at $340 \mathrm{~nm}$. The results represent mean values \pm SEM from 3 independent experiments, which include mean values of 3 technical repetitions. Statistical analyses were performed using two-tailed paired Student $t$-test; ns, not significant.

Figure 4. Transcription of genes $H X K 2$ and $P F K 1$ by deletion of the $S N F 1$ gene. Real-time PCR (qPCR) was performed to measure mRNA levels of $H X K 2$ and PFK1 genes and $U B C 6$ as a reference gene. The results represent mean values \pm SEM from 3 independent experiments, which include mean values of two technical repetitions. Statistical analyses were performed using two-tailed paired Student $t$-test; ns, no significance.

Figure 5. Influence of $S N F 1$ deletion in the mitochondrial respiration. The oxygen consumption was measured at basal state and maximal respiratory capacity in exponential phase. The results represent mean values \pm SEM from 3-5 independent experiments, which includes mean values of 3 technical repetitions. Statistical analyses were performed using two-tailed unpaired Student $t$-test; ns, not significant.

Figure 6. Impact of SNF4 and GAL83 deletion in the mitochondrial respiration. The oxygen consumption was measured at basal state and maximal respiratory capacity in exponential phase. The results represent mean values \pm SEM from 3-5 independent experiments, which includes mean values of 3 technical repetitions. Statistical analyses were performed using two-tailed unpaired Student $t$-test; ns, no significance.

Figure 7. Effect of genes SNF4 and GAL83 on cells growth. The growth curve of $S$. cerevisiae growth with $0.1 \%, 1 \%$, and $10 \%$ glucose. The results represent mean values \pm SEM from four independent experiments, which includes mean values of three technical repetitions. Statistical significance was calculated by one-way ANOVA followed by Dunnet's test $(* P<0.05$ vs. WT; $* * * P<0.0001$ vs. WT; ns=not significant). 


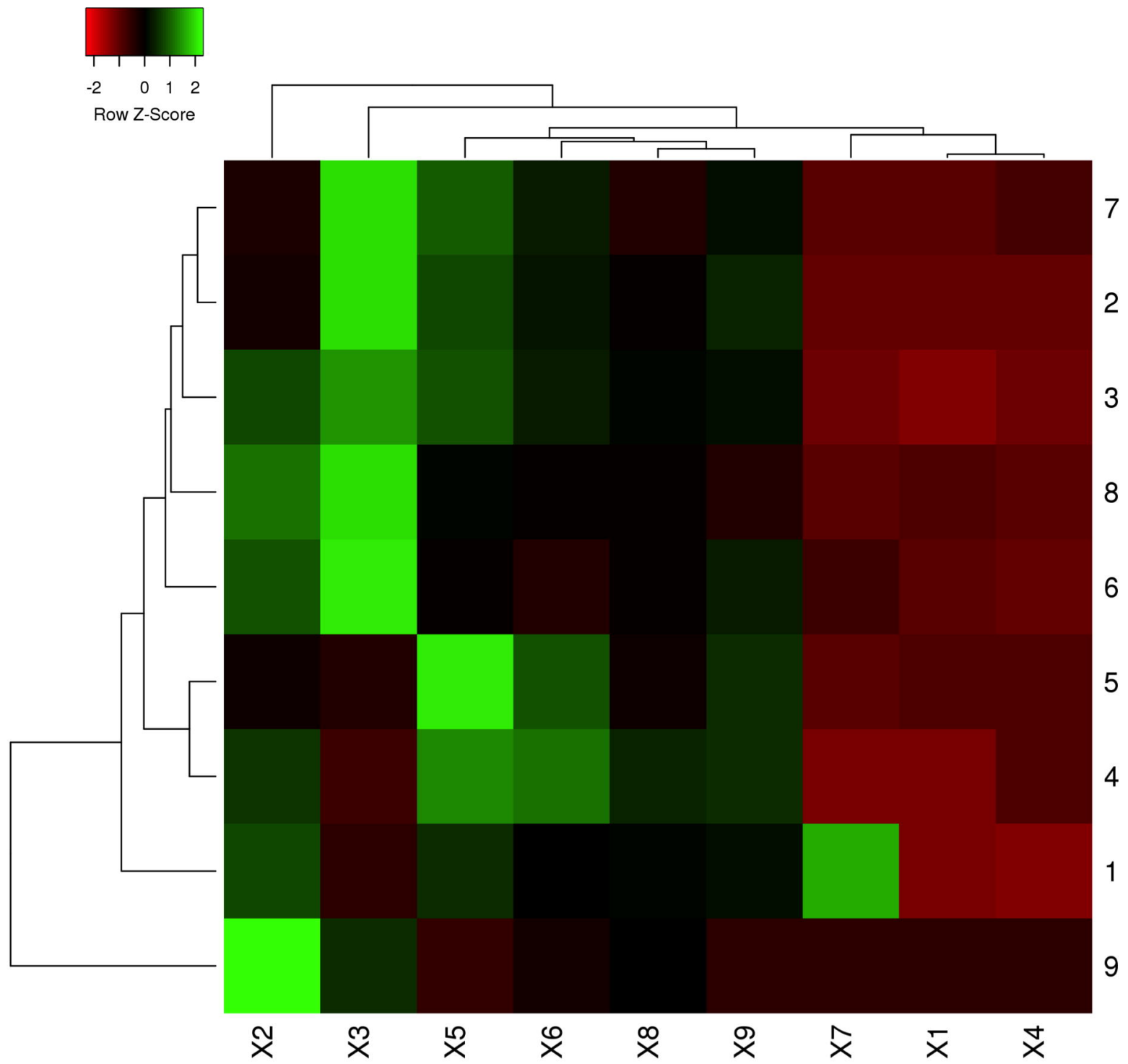

Glucose

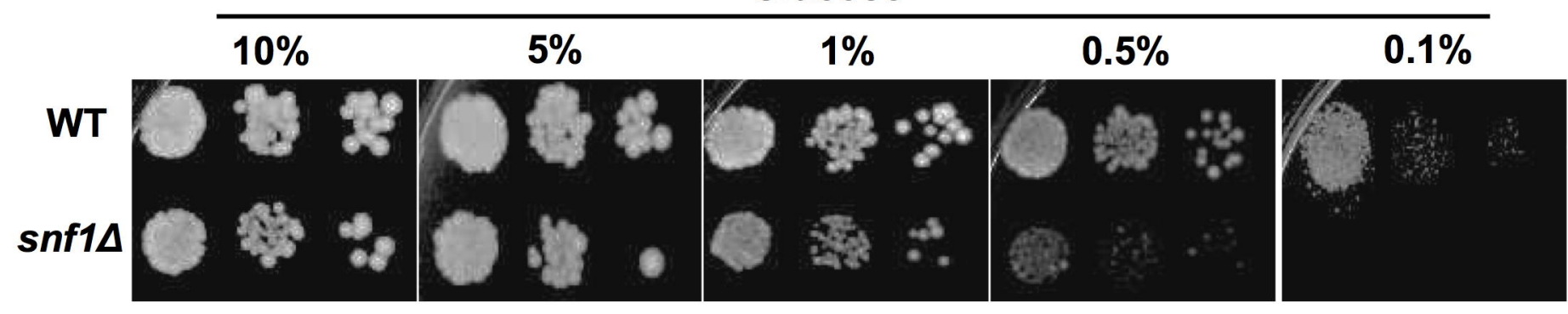




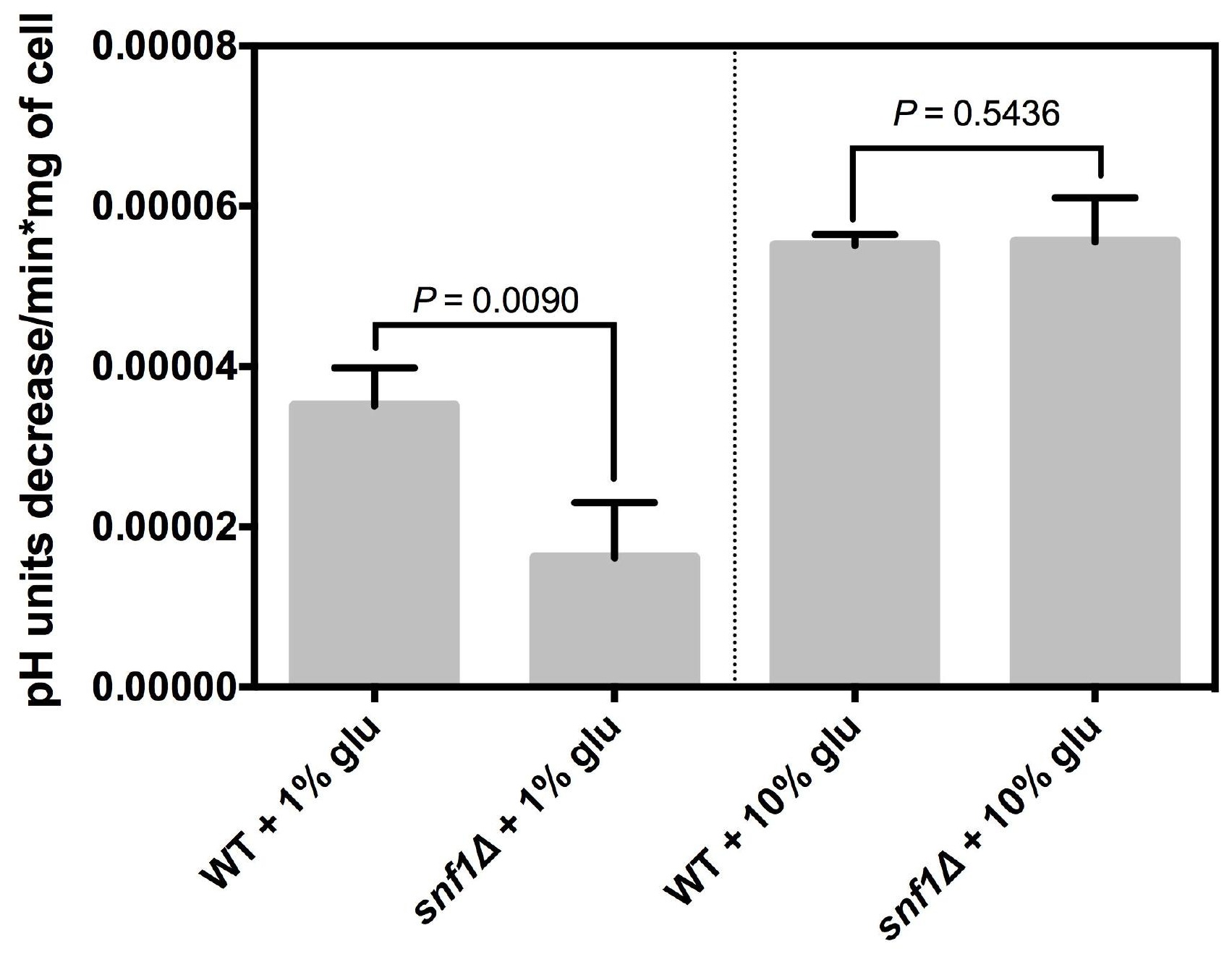



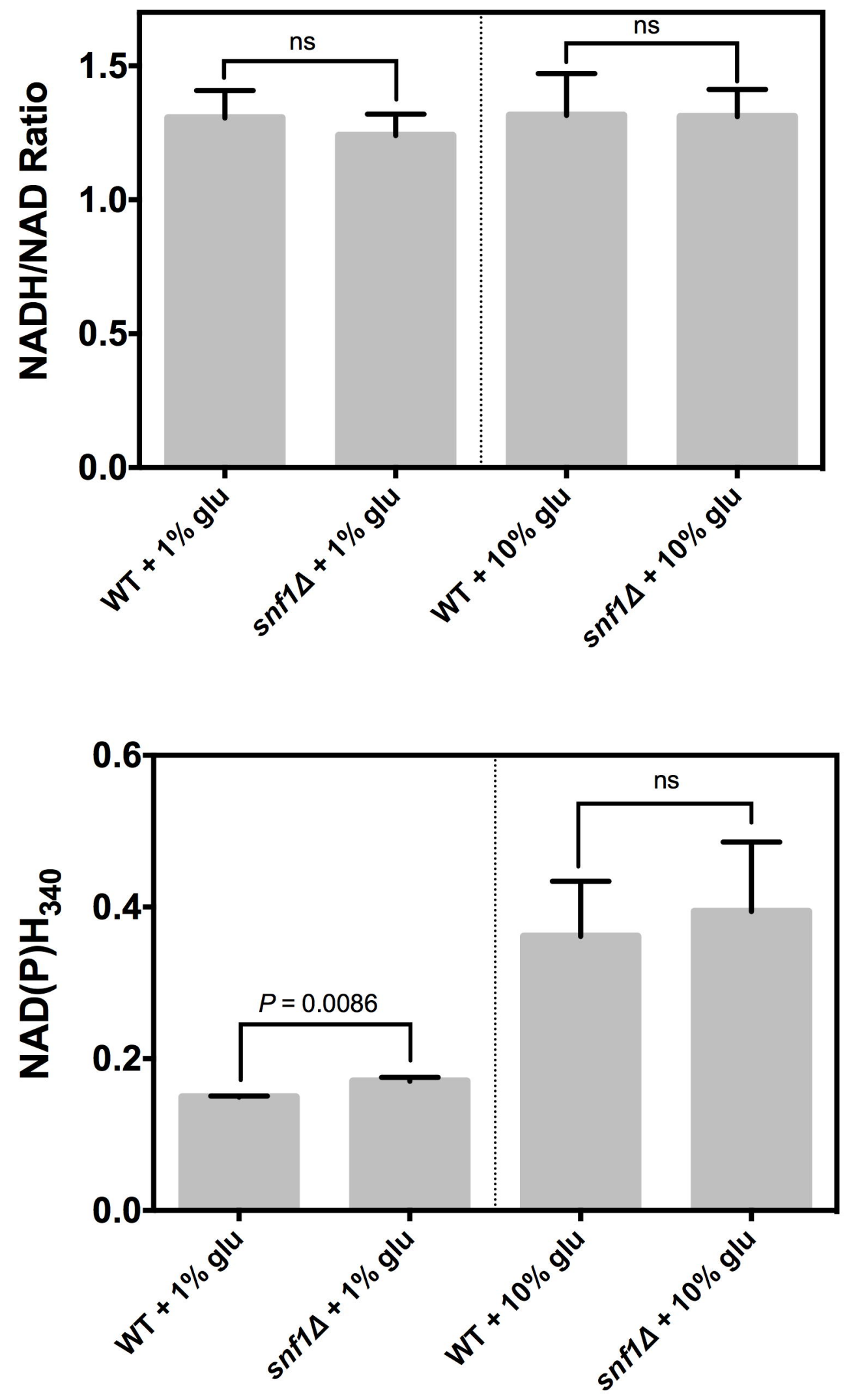

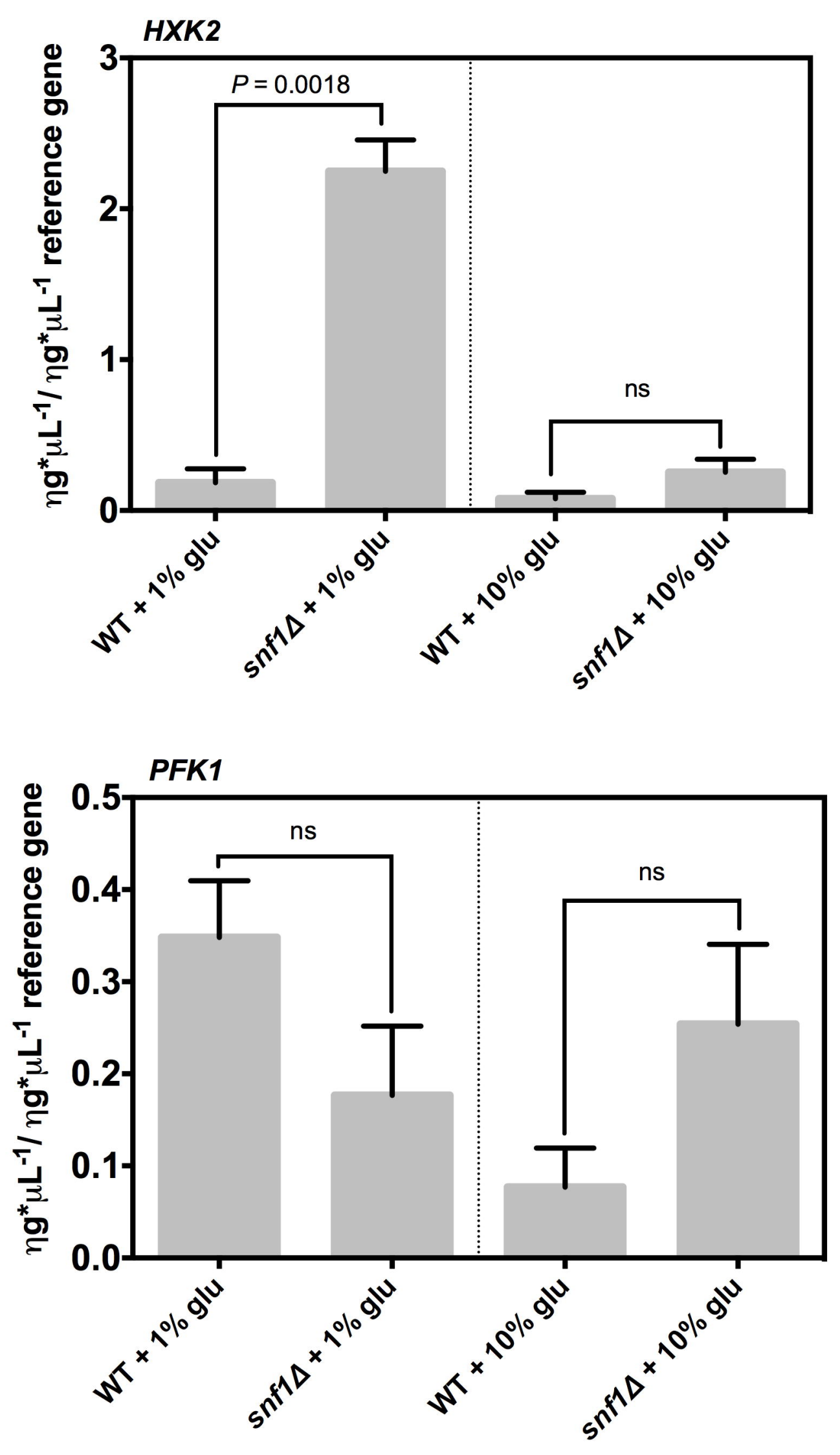
$1 \%$ glucose

$10 \%$ glucose

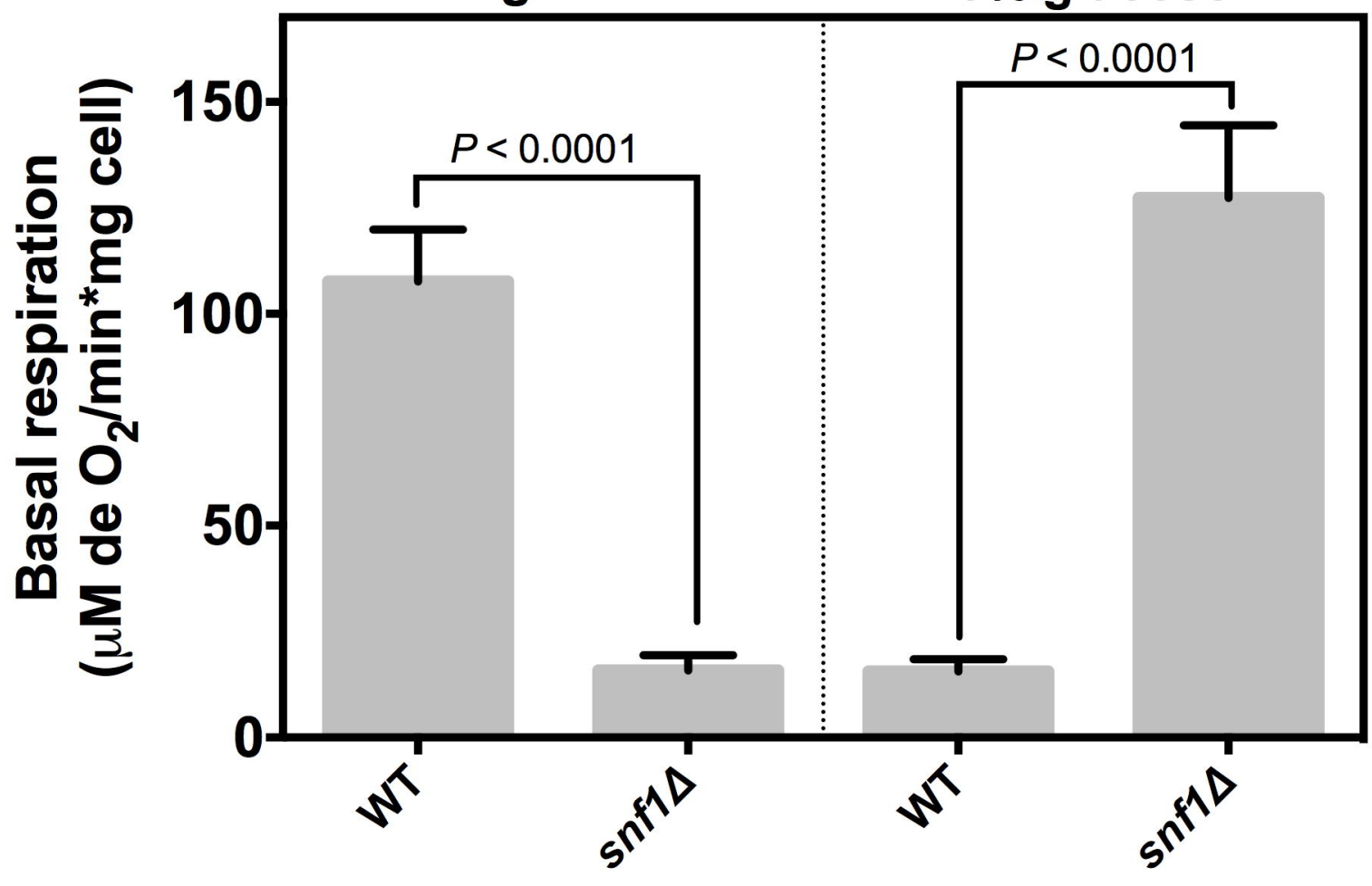

$1 \%$ glucose

$10 \%$ glucose

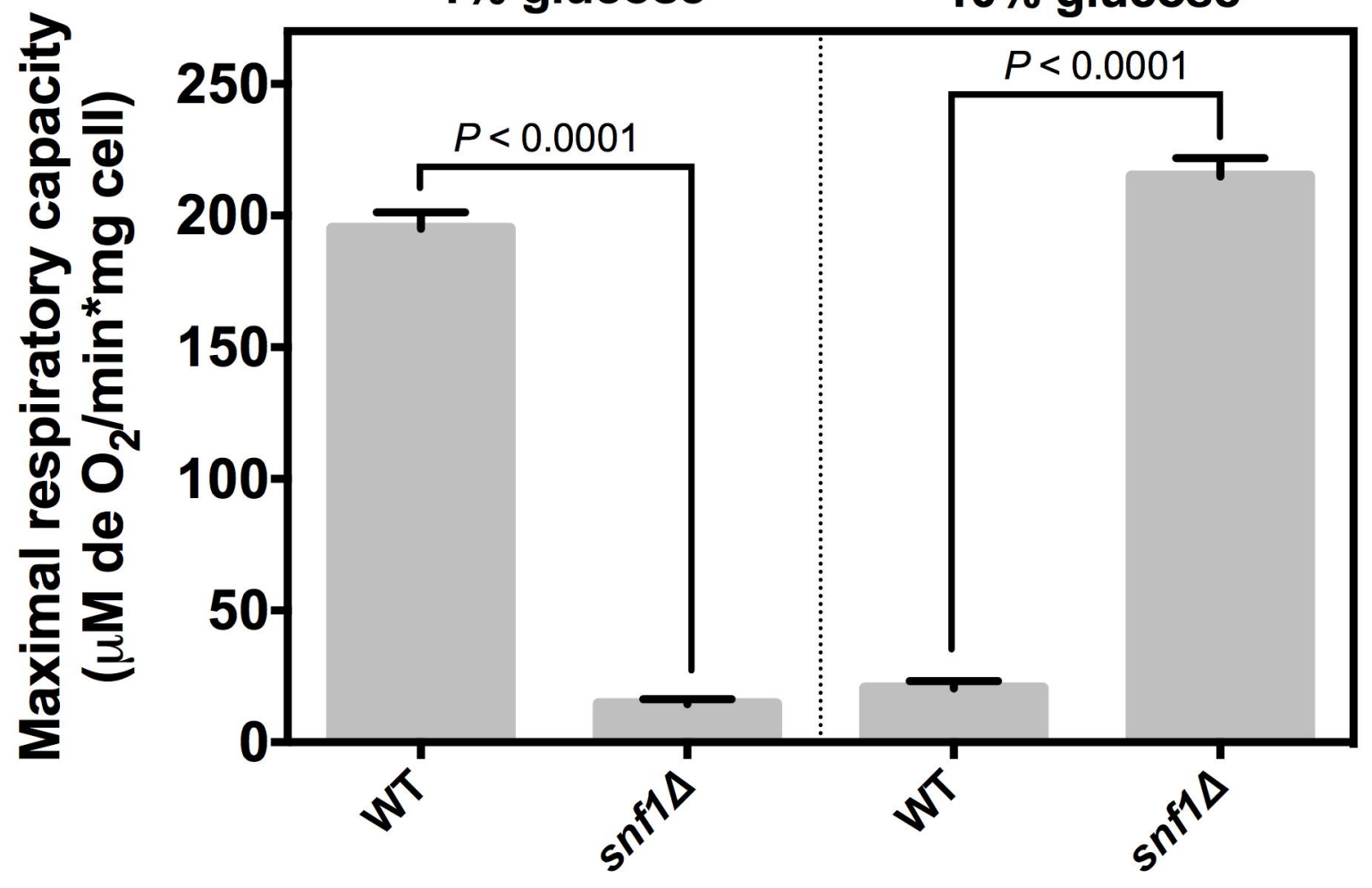


$1 \%$ glucose

$10 \%$ glucose

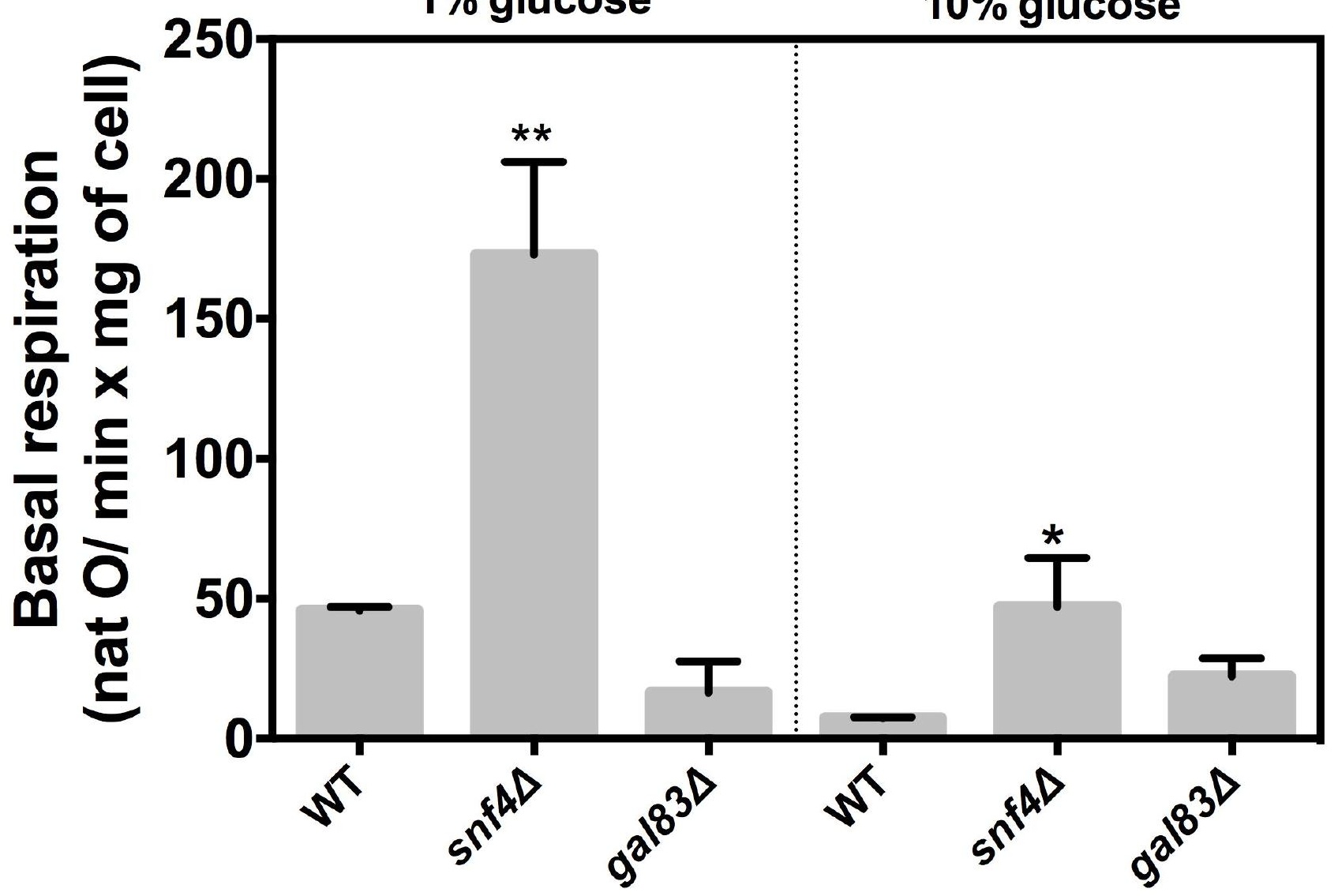


